\title{
LYMPHEDEMA DURATION AS A PREDICTIVE FACTOR OF EFFICACY OF COMPLETE DECONGESTIVE THERAPY
}

\author{
E. Michopoulos, G. Papathanasiou, K. Krousaniotaki, \\ I. Vathiotis, T. Troupis, E. Dimakakos
}

Physiotherapy Department (EM,GP) and Laboratory of Neuromuscular and Cardiovascular Study of Motion-LANECASM (EM,GP,ED), University of West Attica; Department of Internal Medicine, National and Kapodistrian University of Athens, Medical School (KK,IV,TT,ED), Athens, Greece

\section{ABSTRACT}

Lymphedema is a common condition with global impact and a multitude of complications, however, only a few professionals specialize in its management. A retrospective analysis of 105 subjects with unilateral lymphedema upper or lower limb was performed to investigate whether the duration of lymphedema constitutes an important factor associated with the efficacy of complete decongestive therapy (CDT). Subjects were classified into two groups according to the duration of lymphedema, prior to CDT: group $A$ ( $\leq 1$ year) and group $B$ (>1 year). Both groups were treated daily according to the same $C D T$ protocol for four weeks. The CDT efficacy was determined based on the percent reduction of excess volume (PREV) measurements. Lymphedema was significantly reduced in both groups of subjects, but significantly more in group A (p<0.001). In subjects with upper limb lymphedema, median value of PREV was $80.8 \%$ (interquartile range, 79.1-105.0\%) in group $A$ and $62.0 \%$ (interquartile range, 56.7-66.5\%) in group $B(p<0.001)$. In subjects with lower limb lymphedema PREV was $80.7 \%$ (interquartile range, $74.9-85.2 \%$ ) and $64.5 \%$ (interquartile range, 56.0-68.1\%) for groups $A$ and $B$, respectively ( $p<0.001$ ). Duration of lymphedema was found to be a strong predictive factor that may significantly impact CDT efficacy. Therapeutic effects were increased in subjects who were detected and treated earlier for lymphedema.

Keywords: lymphedema, CDT, physical therapy modalities, predictive factor, rehabilitation

Lymphedema is the presence of excess interstitial fluid, high in protein due to insufficient transport capacity of the lymphatic system (1). The lymphatic defect may be due to an error in lymphatic development - termed primary lymphedema, or to an acquired cause by injury to a normal lymphatic system - referred to as secondary lymphedema (2). However, more recent studies have shown that a lymphatic injury is only an initial event, causing several subsequent changes that in some patients lead to the development of secondary lymphedema (3). Primary lymphedema is rare, while secondary type is responsible for the $99 \%$ of individuals with lymphedema; incidence of primary lymphedema is $1 / 100,000$ as opposed to that of secondary type which is estimated at 1/1,000 $(2,4,5)$. The prevalence of lymphedema is usually underestimated, mainly because of the retrospective design of most studies and the fact that they rely on smallsize samples (6).

Regardless of classification, clinical characteristics include chronic swelling, localized 
pain, atrophic skin changes, and secondary skin/soft tissue infections (7). Malignant degeneration also represents a rare yet severe complication of chronic lymphedema (5). According to health-related quality of life questionnaires (HRQoL), lymphedema affects patients' quality of life. There are many factors associated with this effect including, lack of awareness of lymphedema by healthcare professionals, poor information provided to patients, insufficient specialized resources, frequency of acute inflammatory episodes, presence of pain, skin quality, and reduced limb mobility (8).

Although various techniques have been proposed for lymphedema rehabilitation, complete decongestive therapy (CDT) represents the gold standard as the most effective therapeutic approach $(9,10)$. CDT is divided into two phases. The aim of phase I (intensive phase) is to decongest the lymphedematous area, whereas the aim of phase II (maintenance phase) is to optimize and maintain the above results (9). The efficacy and safety of phase I CDT has already been assessed, with the reduction of edema being quite high (11).

From the economic point of view, financial cost of lymphedema is related to direct and indirect costs and increases significantly with increasing stage and severity of lymphedema $(12,13)$. Chronic wounds and cellulitis are mainly responsible for the high costs and disease burden (14). However, despite the significant benefits of CDT in patients with lymphedema, few professionals specialize in its management, leading to substantial delays in detection, misdiagnosis, and inappropriate treatment (15). In addition, lack of reimbursement for treatment adds an extra burden on the delay because of financial pressure it put on patients (12). Duration of lymphedema is empirically considered as an important factor associated with treatment efficacy, but studies that have examined this association are limited and conflicting. Therefore, the connection between duration of lymphedema and CDT efficacy needs further examination since it is of critical importance to public health, not only in Greece, but in many other countries. This is easily understood considering the impact of lymphatic filariasis (more than 129 million patients globally) and iatrogenic injuries as a consequence of surgery, radiation therapy and chemotherapy for cancer (approximately 1 in 5 patients treated for breast cancer will experience lymphedema) $(6,16,17)$.

The purpose of this study is to investigate whether duration of lymphedema composes a potential factor associated with the efficacy of CDT in patients with lymphedema.

\section{MATERIAL AND METHODS}

\section{Study Design and Settings}

A retrospective cohort study of consecutive subjects treated for limb lymphedema according to the principles of CDT was conducted. The database included subjects with lymphedema who were receiving treatment at the Vascular Unit of "Sotiria" General Hospital, Department of Internal Medicine, National and Kapodistrian University of Athens, Athens, Greece between March 2017 and April 2019. The study was conducted on the initiative of researchers from the Laboratory of Neuromuscular and Cardiovascular Study of Motion (LANECASM), University of West Attica.

Medical records and physiotherapy treatment records were retrieved and analyzed. No follow-up data were collected. Written informed consent was obtained from all participants. The study was approved by the Thesis Review Committee of the Institute (Department of Physiotherapy, University of West Attica, issued approval REG NUMBER 1353) (11), conducted in accordance with the Declaration of Helsinki and its later amendments, and reported according to STROBE guidelines (18). Lymphedema measurements and CDT protocol has been described elsewhere (11); details are currently provided in brief.

\section{Study Population}

One hundred and forty-four subjects with lymphedema referred from angiologists, breast surgeons, and oncologists were identified and assessed for enrollment. A thorough history, detailed physical examination, and 
other medical tests including blood tests, chest $\mathrm{X}$-ray, electrocardiography, echocardiogram, colored-flow duplex venous ultrasonography, computer tomography (CT), or lymphoscintigraphy were performed in order to rule out other potential causes of limb edema such as heart or kidney failure, lipedema, etc. (19). The above assessments were completed by a medical physician with expertise in lymphatic diseases in collaboration with an experienced physiotherapist.

Eligible participants had a diagnosis of previously untreated unilateral lymphedema of the limb. Participants were excluded if they had severe heart disease, uncontrolled hypertension, acute lymphangitis, deep vein thrombosis, neuromuscular impairment, and nonadherence to CDT protocol. Finally, 105 subjects fulfilled the criteria and were included in our study (Table 1). The rest (39 subjects, $27 \%$ of the total population assessed) were declined enrollment.

For the purpose of the study, eligible subjects were classified into two distinct groups according to the duration of lymphedema prior to CDT: group A ( $\leq 1$ year) and group $B$ (>1 year).

\section{Complete Decongestive Therapy}

All participants underwent daily CDT. The program was administered 5 days a week for 20 sessions by a specialized physiotherapist, as recommended by the International Society of Lymphology (ISL) (20). Each session included 1 hour of lymphatic massage, also known as manual lymphatic drainage (MLD) with Vodder technique, compression therapy via multilayered, short-stretch (low-stretch) bandages, skin care, and specific lymphedema rehabilitation exercises (9). Furthermore, at each session an assessment was performed to identify issues related to skin infections, such as skin redness, rashes, swelling, heat, itching, and trauma.

The aim of the treatment was to decrease volume of edema and thus improve patient functionality (1). At the end of each treatment instructions were provided to subjects in print and subjects were encouraged to do self-treat- ment (self-lymph drainage, wearing compression garments, doing remedial exercises) and skin care in order to enhance the benefits of CDT $(9,21)$.

\section{Data Collection and Measurements}

Data were collected from each individual patient's clinical records. After a retrospective chart review, the following personal and clinical characteristics were obtained: sex, age, type of lymphedema (primary or secondary), disease stage (I-III), baseline total volume in the affected limb, and edema measurements [excess volume (EV) and percent of excess volume (PEV)].

Type and stage of lymphedema were determined according to the instructions of the consensus document of the ISL (20). Limb measurements were made using a flexible nonstretch tape in combination with validated automated software (Limb Volume Professional, Bioscience Research Institute). EV and PEV were also calculated by the above software. Essentially, EV was defined as the volumetric difference between the affected and unaffected limb (volume in the affected limb volume in the unaffected limb), whereas PEV as the percentage of $\mathrm{EV}$ divided by the volume in the unaffected limb (EV / volume in the unaffected limb) $\times 100$. The response to the therapeutic intervention CDT was estimated by the percent reduction of excess volume (PREV), according to the following formula: [(pre-treatment EV post-treatment EV)/ pretreatment EV] $\times 100$.

Assessments, including EV and PEV measurements, were performed at baseline, end of second week, end of third week, and at the end of therapy. Second and third measurements assessed after completion of 7 days, while last measurement was after 6 days. PEV or relative edema is a more preferable parameter for defining the severity of lymphedema than EV or absolute edema, because of the heterogeneity of the anthropometric characteristics (22). Thus, the classification of severity lymphedema was based on PEV values, distinguishing for the following lymphedema severity categories according to ISL; minimal (PEV: 


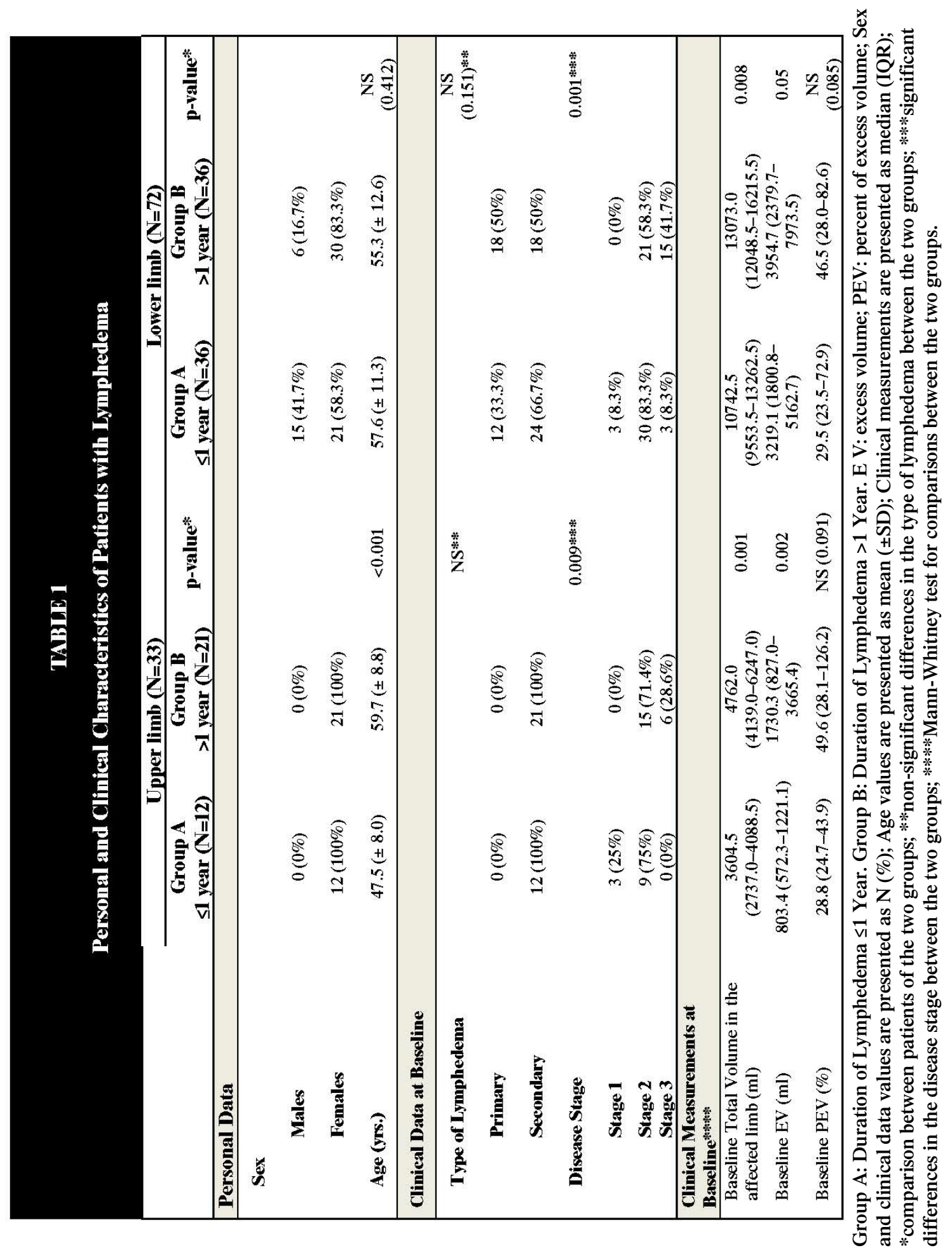




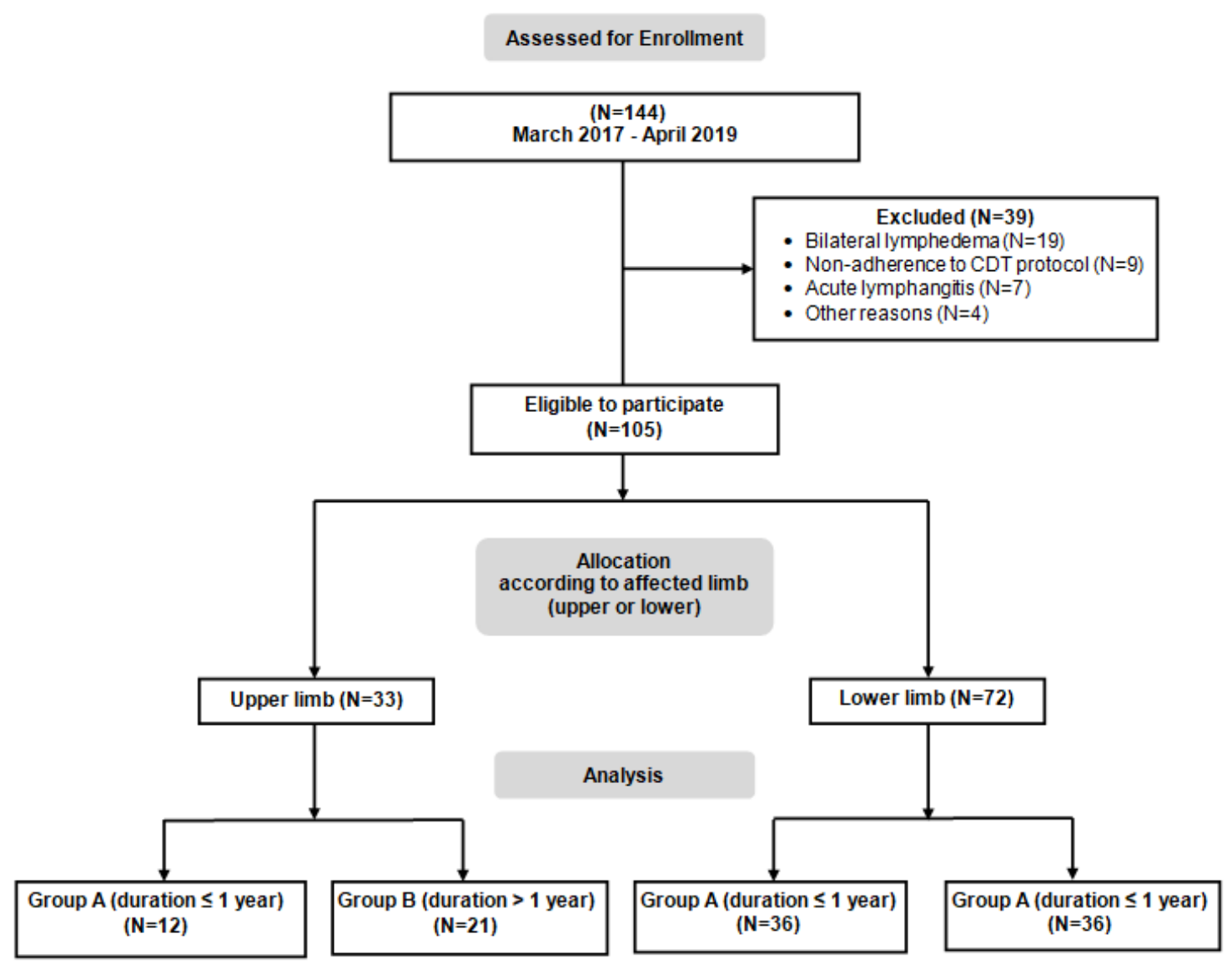

Fig. 1. Flow diagram of the study subjects. CDT: Complete Decongestive Therapy

$>5-<20 \%$ ), moderate (PEV: $20-40 \%$ ) and severe (PEV: $>40 \%$ ). Minimal lymphedema can also be subdivided into; minimal (PEV: $>5$ $10 \%$ ) and mild (PEV: $>10-<20 \%)(20)$.

The short-term efficacy of CDT was evaluated with PREV, which was calculated at the end of CDT (last measurement). PREV was the effective index and the main endpoint of the study. It is known that relative volume change is more reliable and accurate method to quantify lymphedema as opposed to absolute volume change due to the effect of body size on the latter (23). That explains the use of PREV which is the relative value of edema reduction in order to assess the response to therapy. PREV at a value of $100 \%$ implies the return of the affected limb back to normality.

\section{Statistical Analysis}

Statistical analysis of the data was performed using the SPSS v.22 software package. Absolute (N) and relative (\%) frequencies were used to describe categorical variables (sex, type of lymphedema and disease stage). Age values were normally distributed (Kolmogorov-Smirnov test) and are presented as mean \pm standard deviation. Total Volume in the affected limb, EV, PEV and PREV were skewed and are presented as median and interquartile range (IQR $=25$ th-75th percentile values). CDT was defined as the independent variable, while EV, PEV and PREV were the dependent variables examined.

Pearson's chi-squared test $\left(\mathrm{x}^{2}\right)$ for type of 
lymphedema and Fisher's exact test for disease stage were used to examine possible differences between the two groups (Group A: duration of lymphedema $\leq 1$ year, Group B: duration of lymphedema $>1$ year). Differences in age between the two groups were detected by Student's t-test. Non-parametric MannWhitney $U$ test was used to search for significant differences in EV, PEV, and PREV between the two groups. Differences in EV and PEV measurements over time in the same group were screened using analysis of variance (ANOVA). Scatter analysis for repeated measurements was performed using logarithmic transforms. All tests were two-sided at the 0.05 significance level.

\section{RESULTS}

\section{Descriptive Data}

One hundred and forty-four subjects were submitted to CDT intervention for upper and lower limb lymphedema from March 2017 to April 2019 and were assessed for enrollment. Of the 144 subjects, 39 subjects were excluded on the basis of the eligibility criteria. Finally, a total of 105 subjects with unilateral lymphedema were recruited to the study, 48 subjects $(45.7 \%)$ in group $A$ (duration $\leq 1$ year) and 57 subjects $(54.3 \%)$ in group $B$ (duration $>1$ year). A full breakdown of participants' recruitment and the flow-chart of the study can be found in Fig. 1. Subjects' personal characteristics at admission and baseline data are presented in Table 1.

Upper limb lymphedema group consisted of 33 women, all with a secondary type. There were 21 men and 51 women with lower limb lymphedema (women: $58.3 \%$ in group $A$ and $83.3 \%$ in group B). As far as the type of lymphedema in lower limb is concerned, there were both primary and secondary types, but no significant differences were observed between the two groups ( $p=0.151)$. Group A subjects with upper limb lymphedema were significantly younger than group B subjects (means: 47.5 vs. 59.7 years; $p<0.001$ ); however, there were no significant differences with respect to age in subjects with lower limb lymphedema (means: 57.6 vs. 55.3 years; $p=0.412$ ).

In both upper and lower limb lymphedema, subjects of group A had significantly lower disease stage, baseline total volume in the affected limb and EV values compared to group B. Non-significant differences in PEV values were found between the two groups, both for subjects with upper and lower limb lymphedema (Table 1).

\section{Efficacy of CDT}

The change of edema over time according to duration of lymphedema ( $\leq 1$ year and $>1$ year) is summarized in Table 2 and Figs. 2,3 .

EV values were significantly decreased between baseline and last measurement in both groups of subjects ( Table 2, Fig. 2). More specifically, in subjects with upper limb lymphedema, comparing baseline and last measurements demonstrated significantly lower EV values found post-CDT (medians: $803.4 \mathrm{ml}$ vs.

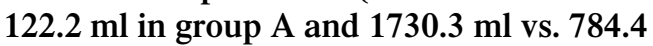
$\mathrm{ml}$ in group $\mathrm{B} ; \mathrm{p}<0.001)$. Subjects with lower limb lymphedema had significant lower EV values at last post-CDT measurements as well (medians: $3219.1 \mathrm{ml}$ vs. $577.1 \mathrm{ml}$ in group A and $3954.7 \mathrm{ml} \mathrm{vs.} 1076.8 \mathrm{ml}$ in group $B$; $\mathrm{p}<0.001$ ).

PEV values were significantly decreased between baseline and last measurement in both groups (Table 2, Fig. 3). In subjects with upper limb lymphedema, significant lower PEV values were found post-CDT (medians: $28.8 \%$ vs. $5.4 \%$ in group $A$ and $49.6 \%$ vs. $24.3 \%$ in group $B ; p<0.001)$. Similar pattern was noted in lower limb as well (medians: $29.5 \%$ vs. $7.6 \%$ in group $A$ and $46.5 \%$ vs. $15.9 \%$ in group $B ; p<0.001$ ).

In the present study, severity of lymphedema in group A was improved from moderate pre-CDT to minimal post-CDT in both upper and lower limbs. An improvement of lymphedema severity, between pre-CDT and post-CDT measurements, was also noted in group B subjects, from severe lymphedema to moderate in upper limb and to mild in lower limb (data not shown). 


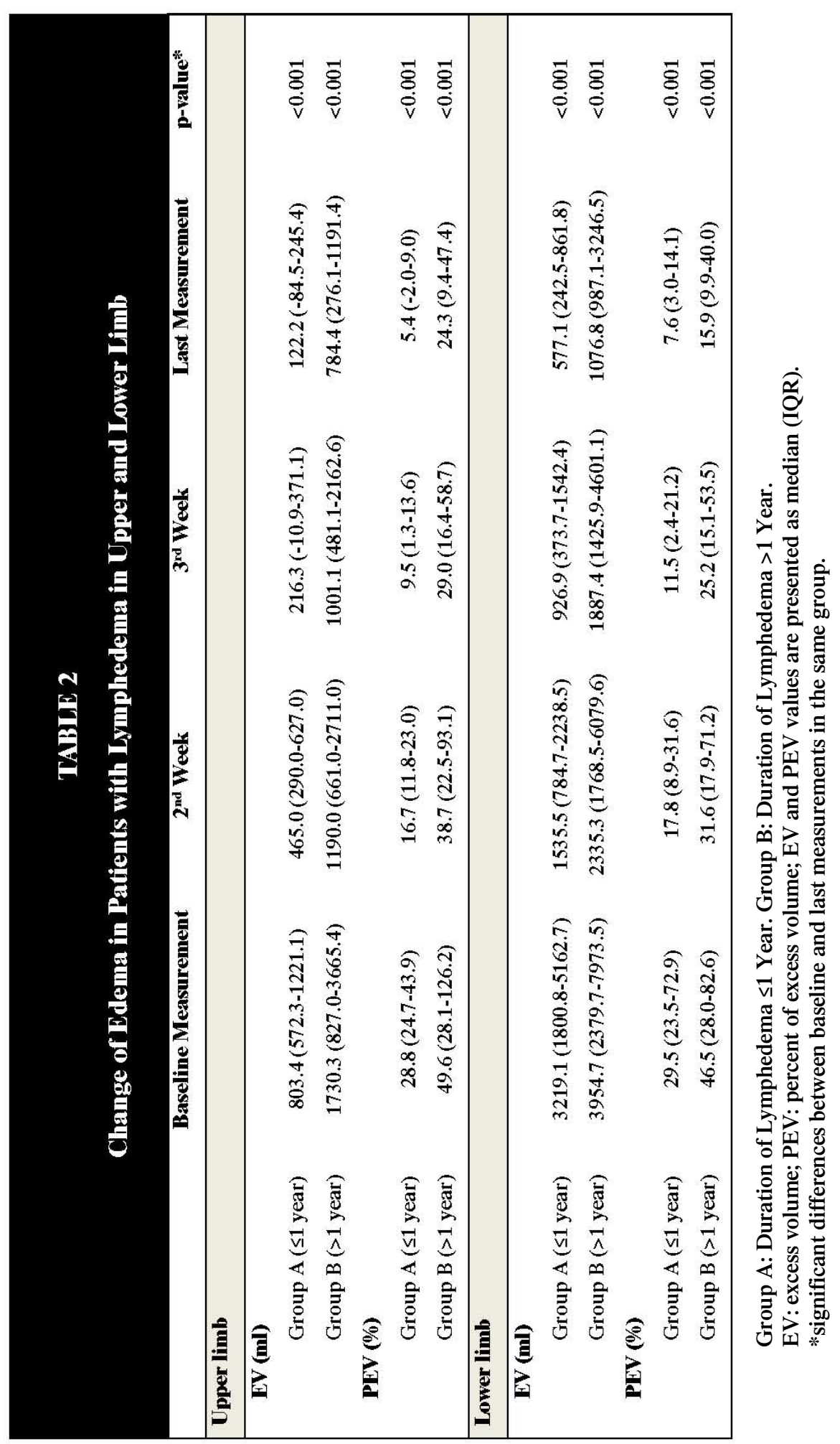




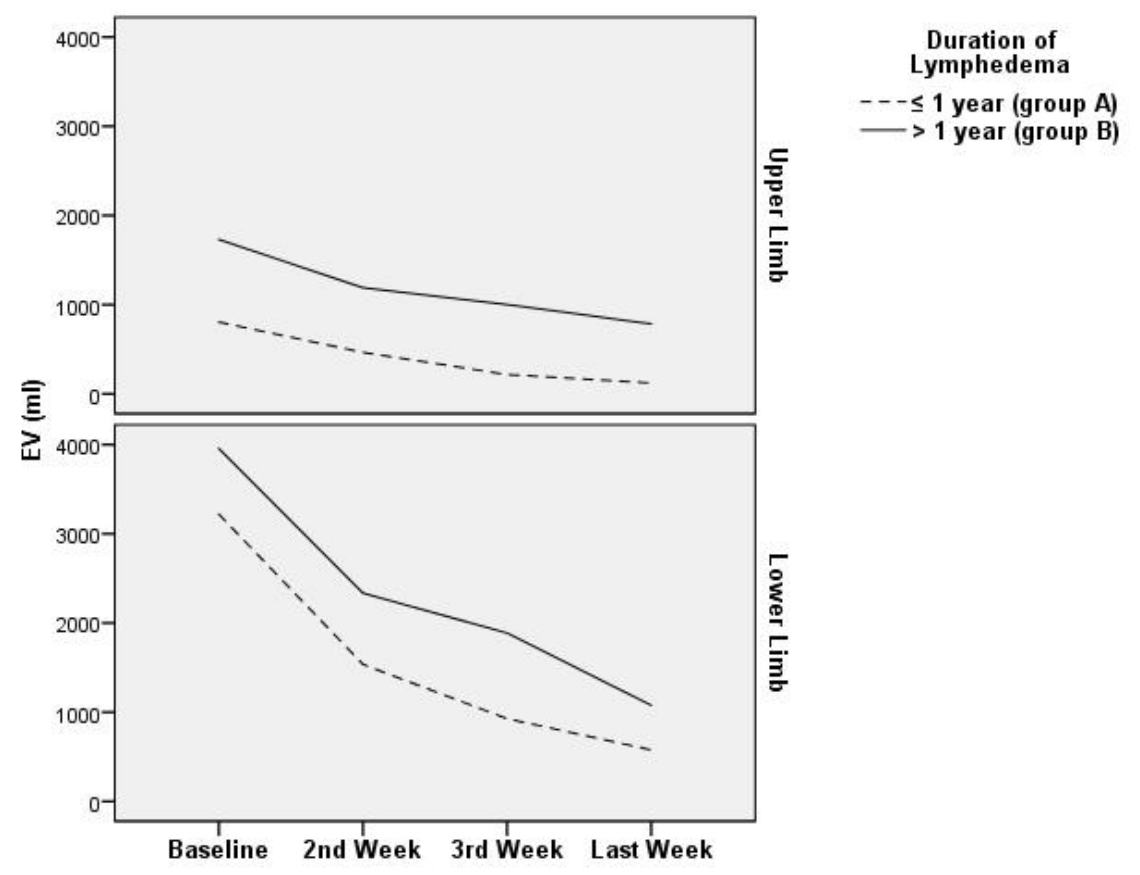

Fig. 2. Changes in Excess Volume (EV) between baseline and weekly measurements in subjects with lymphedema.

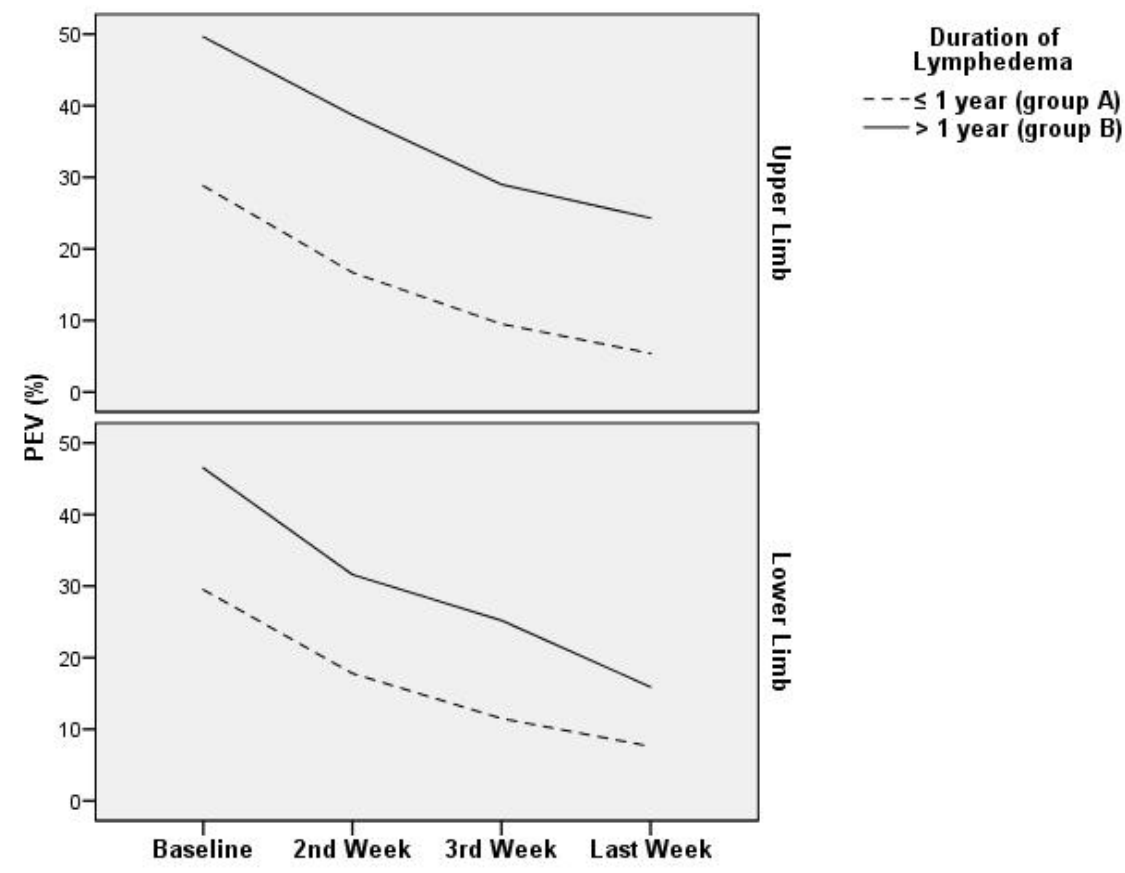

Fig. 3. Changes in Percent of Excess Volume (PEV) between baseline and weekly measurements in subjects with lymphedema. 
TABLE 3

CDT Efficacy based on Percent Reduction of Excess Volume

\begin{tabular}{|lccc|}
\hline & N (\%) & PREV & p-value* \\
\hline Upper limb & & & $<0.001$ \\
Group A ( $\leq 1$ year) & $12(36.4)$ & $80.8(79.1-105.0)$ & \\
Group B (>1 year) & $21(63.6)$ & $62.0(56.7-66.5)$ & $<0.001$ \\
Lower limb & & & \\
Group A ( $\leq 1$ year) & $36(50)$ & $80.7(74.9-85.2)$ & \\
Group B (>1 year) & $36(50)$ & $64.5(56.0-68.1)$ & \\
\hline
\end{tabular}

Group A: Duration of Lymphedema $\leq 1$ Year.

Group B: Duration of Lymphedema $>1$ Year.

CDT: complete decongestive therapy; PREV: percent reduction of excess volume; PREV values are presented as median (IQR).

*Mann-Whitney $\mathrm{U}$ test for comparison of PREV values between the two groups.

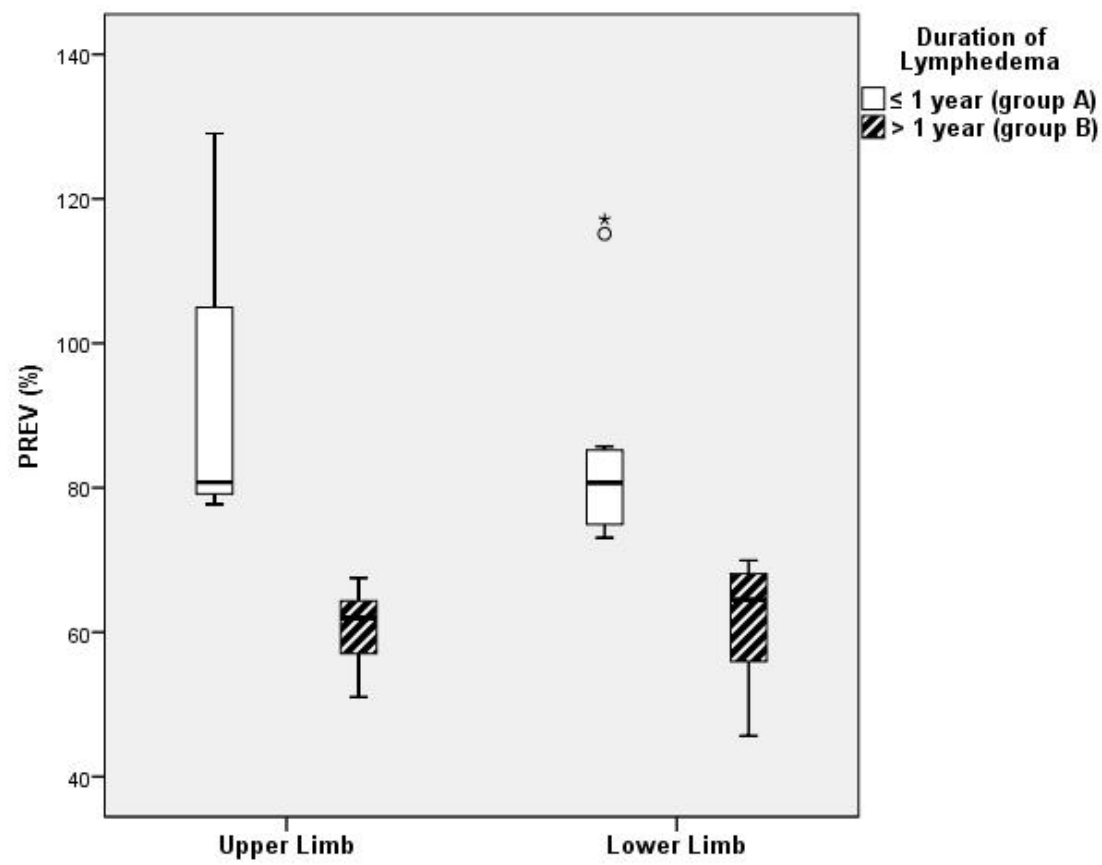

Fig. 4. Differences on CDT Efficacy based on Percent Reduction of Excess Volume (PREV) between groups (Group A: $\leq 1$ year vs. Group B: >1 year), in subjects with lymphedema.

Box: Interquartile range (IQR). Horizontal bar in the box: median value. Whiskers extend from the box: largest and smallest observed values. Circle: Outlier greater than $\pm 1.5 \times I Q R$. Asterisk: Extreme point. 


\section{Duration of Lymphedema}

The effects of duration of lymphedema on CDT efficacy are presented in Table 3 and Fig. 4. PREV values were significantly higher in subjects with time to treatment $\leq 1$ year (group A) compared to those with time to treatment $>1$ year (group B), for both subjects with upper and lower limb lymphedema. PREV median value for subjects with upper limb lymphedema was $80.8 \%$ (interquartile range, 79.1-105.0\%) in group $A$ and $62.0 \%$ (interquartile range, 56.7-66.5\%) in group $B$ $(\mathrm{p}<0.001)$. In subjects with lower limb lymphedema, PREV median value was $80.7 \%$ (interquartile range, 74.9-85.2\%) and 64.5\% (interquartile range, 56.0-68.1\%) in groups $\mathrm{A}$ and $B$, respectively $(\mathrm{p}<0.001)$.

\section{DISCUSSION}

In the present study, duration of lymphedema was found to be an important predictive factor associated with success of CDT treatment as measured by PREV in both upper and lower limbs. Percent reduction of excess volume was significantly higher in subjects with time to treatment $\leq 1$ year compared to those with time to treatment $>1$ year. Lymphedema almost ceases to be visible if CDT is applied early (group A). Nevertheless, in subjects with delayed treatment initiation (group B) lymphedema decreased satisfactorily, but to a significantly lesser extent.

\section{Factors Influencing CDT Efficacy}

Previous studies have indicated that there is no significant correlation between age and CDT efficacy $(22,24-26)$. In contrast, Liao et al (27) reported that age is associated with reduction of lymphedema with older age showing less effective CDT intervention. However, the authors reported that the older subjects had poor bandage compliance. Thus, poor bandage compliance may act as a confounding factor (27). Indeed, bandage compliance has been cited as one of the most important predictors of CDT efficacy and particular attention must be given to properly inform both subjects and family members (22). In our study, subjects received treatment on a daily basis so appropriate bandage application was ensured; we did not encounter any incidents of non-compliance.

Lymphedema staging seems to be a predictive factor of response as CDT is less effective in more advanced stages (26,28-30). Although there are some studies suggesting that stage is not associated with the efficacy of CDT intervention, all studies conclude that CDT should be provided in early stages (22, $24,25)$. Baseline total volume in the affected limb does not appear to affect CDT efficacy $(22,26)$. There also seems to be no correlation between CDT efficacy and type of lymphedema. More specifically, Abakay et al (31) conducted a prospective study enrolling subjects with both types of lymphedema ( 20 subjects in each type) and found that there was no difference in the volume reduction between primary vs. secondary group.

According to previous studies $(22,24,26$, $27,32,33)$ both EV and PEV were found to be significant related with CDT efficacy as measured by PREV. EV and PEV measure absolute and relative edema respectively and are proportional to each other. Haghighat et al (24) found that the baseline volume of edema plays an important role in the effectiveness of treatment in subjects with breast cancer-related lymphedema (BCRL). They claimed that the higher the volume of edema at baseline, the smaller the reduction of lymphedema will be (24). The latter view is also embraced by other studies $(22,26,32,33)$. In line with the above, Forner-Cordero et al (22), Liao et al (27) and Keskin et al (26) have confirmed that higher baseline PEV induces lower PREV. On the contrary, Hwang et al (34) conducted a retrospective chart review and found that subjects with higher PEV at baseline had a greater reduction of lymphedema at the end of CDT. These conflicting results may be due to the fact that in study of Hwang et al (34) group 1 subjects $(\mathrm{PEV}<20 \%)$ had significantly lower mean baseline PEV values compared with the other studies $(22,26,27)$ where lymphedema was moderate. At present, the relationship between PEV and CDT efficacy is not clearly 
delineated and further investigation is needed.

\section{Duration of Lymphedema \& CDT Efficacy}

According to our results, the first year of onset of lymphedema is proposed to be of great importance for the efficacy of CDT treatment in subjects with lymphedema. After that duration of lymphedema, excess volume increases significantly and CDT becomes less beneficial.

Lymphedema is a progressive disease that can lead to chronic inflammation and an accumulation of fibroblasts, keratinocytes, and adipocytes (35-39). Therefore, the duration of lymphedema may be related to a worse treatment outcome, as measured by PREV. According to previous studies, EV tends to increase over time $(40,41)$. This is consistent with our findings where subjects with longer duration of lymphedema had higher baseline EV. Haghighat et al showed that, in addition to the EV prior to the treatment, duration of lymphedema also had a statistically significant effect on CDT efficacy as an independent predictive factor (24).

On the other hand, there are studies that question the effect of lymphedema duration on the effectiveness of CDT as measured by PREV $(22,41,42)$. Vignes et al (41) conducted a study in 357 women with BCRL and found that duration of lymphedema was not significantly associated with the relative reduction of edema and PREV values. Forner-Cordero et al (22) examined potentially predictive factors with response to CDT and found that duration of lymphedema was not associated with the treatment outcome. However, the results of both studies are non-comparable with ours due to the different methodologies followed. Vignes et al set a cut-off point of 2 years for classification of lymphedema duration with $61 \%$ of their subjects having chronic lymphedema for more than 2 years opposed to our study where the cut-off point was set at 1 year and $45.7 \%$ of the study population had lymphedema duration $\leq 1$ year. In the study of Forner-Cordero et al, there was no subgroup classification at baseline with respect to lymphedema duration and the mean duration of lymphedema of their study population was 4 years with $67.8 \%$ of their subjects were classified at stage III.

Liao et al (27) and Keskin et al (26) found that duration of lymphedema was related to PEV. More specifically, they argued that lymphedema duration was positively correlated with PEV, which defines severity of lymphedema. They also claimed that lymphedema duration could only indirectly predict the efficacy of CDT $(26,27)$. In the study of Hwang et al (34), subjects with BCRL were classified into two groups (group 1: $\mathrm{PEV}<20 \%$ and group 2: $P E V \geq 20 \%$ ). In line with the above, it was observed that the duration of lymphedema was significantly higher in group 2 compared to group 1 (medians: 8.5 vs. 27.5 months). On the contrary, in the present study no significant differences were observed in PEV values at baseline between the two lymphedema duration groups. This conflict may be due to the relatively small difference in the duration of lymphedema between the two groups in our study.

\section{Limitations}

The present study is subject to several limitations. First, it is a retrospective cohort study. In addition, Body Mass Index (BMI) was not considered. Vignes et al (43) showed that BMI is a risk factor of developing lymphedema which can also affect its severity. However, according to other studies BMI does not appear to be associated with the efficacy of CDT (measured by relative reduction of edema-PREV) $(22,24,26,41)$. No techniques giving insights into the tissue changes, such as ultrasonography (US), magnetic resonance imaging (MRI), proton MR spectroscopy, or histology were used in the present study in order to evaluate and monitor tissue alterations such as excess adipose tissue and excess fibrosis deposition (35). However, Forner-Cordero et al (22) have indicated that the presence of fibrosis does not represent a predictive factor of CDT efficacy. On the other hand, excess of subcutaneous fat in lymphedema has been indicated to cause a poor response to CDT and likely constitutes a potentially confounding factor (38). Although tissue composition is taken into 
account for the staging of lymphedema, it is essentially based on findings from physical examination (subjective assessment) and does not reflect the distribution of lymphedema (44). More objective and accurate methods are required to precisely delineate disease status $(44,45)$. Future research using objective measures is needed to examine the possible effects of the architectural changes including adipose tissue deposition and fibrosis on CDT efficacy. Another point to consider is that there was no knowledge of subjects' limb dominance. It has been reported that the reduction of lymphedema is significantly higher in subjects with lymphedema in the dominant arm than those in the non-dominant arm (46). When lymphedema is in the non-dominant arm, adipose tissue is increased bilaterally and combined with decrease in lean tissue in the dominant arm. But this was not the case for those with lymphedema in their dominant arm where there was a significant increase only in fat tissue and only in the dominant side (47). However, there are studies indicating no association between arm dominance and CDT efficacy $(22,24)$. Regarding leg dominance, there seems to be minimal effect and thus no limb dominance correction is vital (48). Irrespective of limb dominance, there are two conflicting assumptions around the lymphedematous limb. The first is that lymphedematous limb is commonly used less and thus there is muscle atrophy and probably lymphostasis of the muscles. Also due to resection of tissue or volume contraction due to fibrosis, the affected limb can be smaller than the unaffected limb post-CDT $(46,49)$. On the other hand, Brorson et al (50) reported increased amounts of muscle and bone tissue in lymphedematous arm and they attributed it to higher mechanical load on both the muscle and the skeleton. This issue goes beyond the scope of the present study, but it remains necessary to be explored in the future.

\section{CONCLUSION}

In conclusion, according to the findings of this study duration of lymphedema was found to be a strong predictive factor that may significantly enhance the CDT efficacy in individuals with upper and lower limb lymphedema. Percent reduction of excess volume was significantly higher in subjects with time to treatment $\leq 1$ year compared to those with time to treatment $>1$ year. The combination of early diagnosis and treatment is the key to success in lymphedema rehabilitation.

\section{CONFLICT OF INTEREST AND DISCLOSURE}

All authors declare that no competing financial interests exist.

\section{REFERENCES}

1. Bittar, S, R Simman, F Lurie: Lymphedema: A practical approach and clinical update. Wounds 32 (2020), 86-92.

2. Schook, CC, JB Mulliken, SJ Fishman, et al:Primary lymphedema: Clinical features and management in 138 pediatric patients. Plast. Reconstr. Surg. 127 (2011), 2419-2431

3. Kataru, RP, I Wiser, JE Baik, et al: Fibrosis and secondary lymphedema: Chicken or egg? Transl. Res. 209 (2019), 68-76.

4. Smeltzer, DM, GB Stickler, A Schirger: Primary lymphedemas in children and adolescents: A follow-up study and review. Pediatrics 76 (1985), 206-218.

5. Greene, AK, A Slavin, H Brorson (Eds): Lymphedema: Presentation, Diagnosis, and Treatment. 1stEdition. Springer, Switzerland, 2015.

6. Rockson, SG, KK Rivera: Estimating the population burden of lymphedema. Ann. N. Y. Acad. Sci. 1131 (2008), 147-154.

7. Basta, MN, LL Gao, LC Wu: Operative treatment of peripheral lymphedema: A systematic meta-analysis of the efficacy and safety of lymphovenous microsurgery and tissue transplantation. Plast. Reconstr. Surg. 133 (2014), 905-913.

8. Morgan, PA, PJ Franks, CJ Moffatt: Healthrelated quality of life with lymphoedema: A review of the literature. Int. Wound. J. 2 (2005), 47-62.

9. Tzani I, M Tsichlaki, E Zerva, et al: Physiotherapeutic rehabilitation of lymphedema: State-of-the-art. Lymphology 51 (2018), 1-12.

10. Gloviczki, P, MC Dalsing, B Eklöf, et al 
(Eds): Handbook of Venous and Lymphatic Disorders. Guidelines of the American Venous Forum. 4th Edition. CRC Press, Boca Raton, 2017.

11. Michopoulos E, G Papathanasiou, G Vasilopoulos, et al: Effectiveness and safety of complete decongestive therapy of phase I: A lymphedema treatment study in the Greek population. Cureus 12 (2020), e9264.

12. Boyages, J, Y Xu ,S Kalfa, et al: Financial cost of lymphedema borne by women with breast cancer. Psychooncology 26 (2017), 849855.

13. Stout NL, LA Pfalzer, B Springer, et al: Breast cancer-related lymphedema: Comparing direct costs of a prospective surveillance model and a traditional model of care. Physic. Ther. 92 (2012), 152-163.

14. Gutknecht M, K Herberger, K Klose, et al: Cost-of-illness of patients with lymphoedema. J. Eur. Acad. Dermatol. Venereol. 31 (2017),1930-1935

15. Schulze H, M Nacke, C Gutenbrunner, et al: Worldwide assessment of healthcare personnel dealing with lymphoedema. Health. Econ. Rev. 8 (2018), 1-11.

16. Garza R 3rd, R Skoracki, K Hock, et al: A comprehensive overview on the surgical management of secondary lymphedema of the upper and lower extremities related to prior oncologic therapies. BMC Cancer 17 (2017), 468.

17. DiSipio T, S Rye, B Newman, et al: Incidence of unilateral arm lymphoedema after breast cancer: A systematic review and metaanalysis. Lancet Oncol. 14 (2013), 500-515.

18. Von Elm, E, DG Altman, M Egger, et al: The Strengthening the Reporting of Observational Studies in Epidemiology (STROBE) statement: Guidelines for reporting observational studies. Lancet 370 (2007), 1453-1457.

19. Borman, P: Lymphedema diagnosis, treatment, and follow-up from the view point of physical medicine and rehabilitation specialists. Turk. J. Phys. Med. Rehabil. 64 (2018), 179-197.

20. International Society of Lymphology: The diagnosis and treatment of peripheral lymphedema: 2020 consensus document of the International Society of Lymphology. Lymphology 53 (2020), 3-19.

21. Lu, SR, RB Hong, W Chou, et al: Role of physiotherapy and patient education in lymphedema control following breast cancer surgery. Ther. Clin. Risk. Manag. 11 (2015), 319-327.

22. Forner-Cordero, I, J Muñoz-Langa, A Forner-Cordero, et al: Predictive factors of response to decongestive therapy in patients with breast-cancer-related lymphedema. Ann. Surg. Oncol. 17 (2010), 744-751.

23. Ancukiewicz M, CL Miller, MN Skolny, et al: Comparison of relative versus absolute arm size change as criteria for quantifying breast cancer-related lymphedema: The flaws in current studies and need for universal methodology. Breast Cancer Res. Treat. 135 (2012), 145-152.

24. Haghighat, S, M Lotfi-Tokaldany, AA Khadem Maboudi, et al: Predictive factors of response to phrase I complete decongestive therapy in upper extremity lymphedema following breast carcinoma in Iran. Lymphology 46 (2013), 97-104.

25. Mondry, TE, RH Riffenburgh, PA Johnstone: Prospective trial of complete decongestive therapy for upper extremity lymphedema after breast cancer therapy. Cancer J. 10 (2004), 42-48.

26. Keskin, D, M Dalyan, S Ünsal-Delialioglu, et al: The results of the intensive phase of complete decongestive therapy and the determination of predictive factors for response to treatment in patients with breast cancer related lymphedema. Cancer Reports 3 (2020), e1225.

27. Liao, SF, SH Li, HY Huang, et al: The efficacy of complex decongestive physiotherapy (CDP) and predictive factors of lymphedema severity and response to CDP in breast cancer-related lymphedema (BCRL). Breast 22 (2013), 703-706.

28. Lawenda, BD, TE Mondry, PA Johnstone: Lymphedema: A primer on the identification and management of a chronic condition in oncologic treatment. CA Cancer J. Clin. 59 (2009), 8-24.

29. Leal, NF, HH Carrara, KF Vieira, et al: Physiotherapy treatments for breast cancerrelated lymphedema: a literature review. Rev. Lat. Am. Enfermagem 17 (2009), 730-736.

30. Strossenreuther, R, I Dax, C Emde: Lymphedema-treatment. MMW Fortschr. Med. 146 (2004), 28-30.

31. Abakay, H, H Doğan, HT Çaliş, et al: Is the Effect of complex decongestive therapy the same for primary and secondary lower lymphedema? Lymphat. Res. Biol. (2020), 165-174 
32. Ramos, SM, LS O'Donnell, G Knight: Edema volume, not timing, is the key to success in lymphedema treatment: Am. J. Surg. 178 (1999), 311-315.

33. Forner-Cordero, I, J Muñoz-Langa, JM DeMiguel-Jimeno, et al: Physical therapies in the treatment of lymphedema: Preliminary results of a phase III, multicenter, randomized, double-blind, controlled study. Abstracts of the XXXIV ESL Congress, June 25-27, Naples, Italy. Eur. J. Lymphol. Relat. Probl. 19 (2008), 6.

34. Hwang, JM, JH Hwang, TW Kim, et al: Long-term effects of complex decongestive therapy in breast cancer patients with arm lymphedema after axillary dissection. Ann. Rehabil. Med. 37 (2013), 690-697.

35. Fumiere, E, O Leduc, $S$ Fourcade, et al: MR imaging, proton MR spectroscopy, ultrasonographic, histologic findings in patients with chronic lymphedema. Lymphology 40 (2007), 157-162.

36. Norman, SA, AR Localio, SL Potashnik, et al: Lymphedema in breast cancer survivors: Incidence, degree, time course, treatment, and symptoms. J. Clin. Oncol. 27 (2009), 390397.

37. Johnson, KC, M DeSarno, T Ashikaga, et al: Ultrasound and clinical measures for lymphedema. Lymphat. Res. Biol. 14 (2016), 8-17.

38. Brorson, $\mathrm{H}, \mathrm{K}$ Ohlin, G Olsson, et al: Adipose tissue dominates chronic arm lymphedema following breast cancer: An analysis using volume rendered CT images. Lymphat. Res. Biol. 4 (2006), 199-210.

39. Olszewski, WL, M Zaleska, M Cakala: Lymphedema is more than excess of fluid: A lympho-fibro-adipo-edema. Veins and Lymphatics 7 (2018), 7984.

40. Vignes, S, M Arrault, A Dupuy: Factors associated with increased breast cancerrelated lymphedema volume. Acta. Oncol. 46 (2007), 1138-1142.

41. Vignes, S, R Porcher, A Champagne, et al: Predictive factors of response to intensive decongestive physiotherapy in upper limb lymphedema after breast cancer treatment: A cohort study. Breast Cancer Res. Treat. 98 (2006), 1-6.
42. Yamamoto, R, T Yamamoto: Effectiveness of the treatment-phase of two-phase complex decongestive physiotherapy for the treatment of extremity lymphedema. Int. J. Clin. Oncol. 12 (2007), 463-468.

43. Vignes, S, R Porcher, M Arrault, et al: Factors influencing breast cancer-related lymphedema volume after intensive decongestive physiotherapy. Support Care Cancer 19 (2011), 935-940.

44. Suehiro, K, N Morikage, M Murakami, et al: Significance of ultrasound examination of skin and subcutaneous tissue in secondary lower extremity lymphedema. Ann. Vasc. Dis. 6 (2013), 180-188.

45. Johnson, KC, AG Kennedy, SM Henry. Clinical measurements of lymphedema. Lymphat. Res. Biol. 12 (2014), 216-221.

46. Sitzia, J, M Woods, P Hine, A Williams, et al: Characteristics of new referrals to twentyseven lymphoedema treatment units. Eur. J. Cancer Care (Engl) 7 (1998), 255-262.

47. Dylke, ES, LC Ward, JD Meerkin, et al: Tissue composition changes and secondary lymphedema. Lymphat. Res. Biol. 11 (2013), 211-218.

48. Ward, LC, E Dylke, S Czerniec, et al: Reference ranges for assessment of unilateral lymphedema in legs by bioelectrical impedance spectroscopy. Lymphat. Res. Biol. 9 (2011), 43-46.

49. Morgan, RG, JR Casley-Smith, MR Mason, et al: Complex physical therapy for the lymphoedematous arm. J. Hand Surg. Br. 17 (1992), 437-441.

50. Brorson, H, K Ohlin, G Olsson, et al: Breast cancer-related chronic arm lymphedema is associated with excess adipose and muscle tissue. Lymphat. Res. Biol. 7 (2009). 3-10.

Evangelos Dimakakos, MD, PhD, EDA/VM, MLD/CDT

Department of Internal Medicine

University of Medicine, Athens

Sotiria General Hospital, Athens

Aisopou 10 Marousi

Athens, Greece 15122

Tel: 00306972000842

Email: edimakakos@yahoo.gr 